\title{
Solidification of hazardous waste as a part of the raw material recovery process
}

\author{
Daria Polek $^{1, *}$ \\ ${ }^{1}$ Department of Environmental Engineering, AGH University of Science and Technology, Kraków, Mickiewicza 30, 30-059, Poland
}

\begin{abstract}
Paper presents the process capabilities for solidification hazardous waste. In the first part of the article the authors present general technologies and methods in a comparative model. The following section describes the results of market research for the most advanced and innovative solidification technologies. Comparative analysis of the material has shown and described the three most promising, leading-edge technologies of waste solidification avalible on the Polish market.
\end{abstract}

\section{Introduction}

Solidification of waste also referred as chemical immobilization, is the process of waste disposal using their physicochemical properties $[1,5]$.

The main idea is to bring a waste into a form, which is as neutral as it would be possible to the natural environment [3]. Successively carried out could be a process, which makes that waste:

- maximally sorption;

- increases its mechanical strength;

- increases its frost resistance.

Noticeable is the fact that the process of waste immobilization counteracts the leaching of harmful to the environment soluble metal compounds such as:

- ashes and slags from thermal processes;

- industrial dusts and muds;

- waste from the chemical industry;

- waste from galvanic processes.

Solidification is currently one of the most popular methods of waste disposal for inorganic [4] (or with a small admixture of organics) hazardous waste contained in lands and sludges. It also serves for the treatment of sewage sludge contaminated with heavy metals.

Solidification technology was developed and successively implemented in the United States for the process of hazardous waste disposal. The U.S. EPA (Environmental Protection Agency) defines this technology as the best available technology (BAT) for the disposal of about 60 types of hazardous waste.

\section{Methods of hazardous waste immobilization}

Modern technological processes of waste immobilization are carried out in fully automated mixers, which include hydraulic binder (eg lime, cement), water, solidifying chemicals and optional fillers (eg sand, gravel, slag) as an addition for main substratum. In an immobilization processes comprehensive chemical waste analysis is crucial because selected chemicals and the composition of the final solidification mixture are based on its results. The minimal effect that could be obtained after the immobilization process is that the material would be no longer characterized as hazardous and becomes an inconsistent or non-hazardous waste. Very often there is a simultaneous possibility to improve the physical waste properties in order to meet the requirements for reuse in the scope of:

- road construction;

- general and industrial construction (blocks, fittings, concrete products);

- raw meal components for cement plants (substitute for alumina, iron, calcium, and silicon);

- inert material for the landfills (seals, slope profiling, reclamation, recultivation) [2].

Currently, there are four dominant technologies for solidification municipal and hazardous waste that are briefly described below.

\subsection{Phoenix Ash TechnologyColour illustrations}

It is a solidification process in which a mixture of fly ash, volcanic dust, and other solid materials are used to produce bricks and other concrete mixes. This is an example of the method of neutralizing pollutants using cement, which reduces the leaching of impurities from the resulting product. The process is carried out at ambient temperature and involves a significant reduction on waste volume and improving its strength. Technology could be applied to all types of waste (including hazardous and radioactive). PAT technology is one of the first pollution immobilization technologies that has received BAT certification in the USA. The average cost of waste neutralization without additional preparation is estimated at $\$ 60 / \mathrm{Mg}$.

\footnotetext{
* Corresponding author: polek@agh.edu.pl
} 


\subsection{Solidification process with Portland cement}

The second group of waste immobilization technologies is the solidification process with Portland cement [6]. Portland cement is produced on the basis of Portland clinker with a small addition of gypsum. The main Portland clinker minerals are di- and tricalcium silicate (alite and belite), which constitute together $74 \%$ by weight.

This way is the best known and refined method of solidification for all types of waste and has become the basis for many individual technological solutions such as mixtures with other mineral binding substratums, like fly ash, blast furnace slag, roasted or hydrated lime. The undisputed advantage of this technology is the ability to use it in a variety of environmental conditions, both in stationary installations and on mobile devices. The average cost of that method is about $\$ 40 / \mathrm{Mg}$.

\subsection{Encapsulation method}

Another group of solidification methods is related to the use of sulfur in the polymerization processes (so-called encapsulation). This technology based on mixing material with sulfur waste at a temperature of about $120^{\circ} \mathrm{C}$ to form a durable polymer bonds. The use of this method is particularly recommended for waste with high chemical (acidic and alkaline) aggressiveness. Comparing to conventional methods, the use of sulfur in the waste disposal process could reduce its cost-effectiveness by about four times (comparing to conventional cementbased technologies).

The encapsulation process could also be used as an additional barrier to the migration of toxic impurities from solidified wastes in hydraulic matrices by covering them with that particular product.

\subsection{Polymerization}

The last technology, which is using for permanently binding waste materials, is their polymerisation using chemical compounds. This is the latest technology group that is constantly evolving. Similarly to the sulfuric method, the high temperature of the process is important. In addition, it is necessary to ensure the consistency of the composition of the waste stream. This means carrying out costly chemical analyzes of each batch of the batch. The cost of using this technology is almost twice as expensive than the use of sulfur or cement and is therefore relatively rarely used in large-scale industries.

\section{Market analysis of available technologies}

The enormous diversification of the hazardous waste which could be solidified and the dynamically changing waste market have led to the rapid development and patenting of many new technological variants of the above-mentioned immobilization methods. According to a global market analysis, most solid waste companies have their own unique technologies. Each of the technologies are used under protection by appropriate patents and licensing rights and in many cases they are not a subject of sale and there is no possibility for further modifications. According to these reasons, in the article, authors decided to show three most advanced technologies offered for sale on the Polish market (with the possibility of adapting them to the individual investor's order).

\subsection{GEODUR Technology}

Geodur technology is based on patents EP 1200158 B1 and PL 192267. It is based on the appropriate stoichiometric selection of individual solidifying reagents (mainly acids and alkalis). During the waste immobilization using the Geodur system, it comes to the chemical transformation of material ("internal barriers") and physical sealing ("external barriers") and achieved physical parameters (minimal compressive strength, limited water permeability). Components of GEODUR products are readily soluble. Monomeric and polymeric substances activate the bonding properties of the added binders, such as cement, lime or fly ashes, by varying their surface tension. Components of GEODUR products create with harmful substances complex compounds or directly molecular substances. GEODUR products simultaneously act as a solvent for various components of the mix. Depending on the type of impurities contained in the immobilized waste, additional are generally used inorganic additives and binders.

One of the most important mechanisms is to change the $\mathrm{pH}$ value. This is possible by the addition of pozzolanic binders - the most often fly ash or lime. In addition to changing the $\mathrm{pH}$ of the waste to about 9-11, the accompanying element is the precipitation of heavy metals in the form of hydroxides. There is also a change in validity during the process, which is based on reducing the valence of impurities by inorganic additives such as $\mathrm{FeSO}_{4}$.

Another significant mechanism is achived through insoluble mercaptans compounds in the formation insoluble complex compounds from lead and zinc.

If a waste immobilization product should be used as construction material, it is suggested, to place additional physical process that includes and change, in particular, density, surface change and compression resistance. It is also important to increase the density of the treated waste by adding cement or fly ash and adding to the production process energy mix. Accompanying factors, besides changing the density, are also the compressive strength, change of surface and permeability. Compression resistance is necessarily associated with the use of cement or pozzolanic reactive waste, which may be, for example, wastes from the combustion of sludge from a treatment plant containing calcium.

The process also has a direct impact on the consistency of waste. Depending on the moisture of the mixture and the energy supplied, solidified and stabilized material of different consistency is formed (monoliths, granulates). Pellets are produced at the highest energies transferred into a process. 
The decision on which chemical additives to use in the process depends directly on the stoichiometric calculations. This is a specially adapted GEOCALC ${ }^{\circledR}$ computer program and GEODAT ${ }^{\circledR}$ data bank. There are currently over 2,300 recipes in the GEODAT ${ }^{\circledR}$ data bank for approximately 730 different types of waste.

The production process of the installation could be managed comprehensively (via internet connection) in GEOSYS ${ }^{\circledR}$ system using the above database and software. The installation control system is compatible

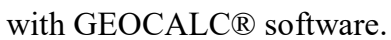

Currently, Geodur technology is applied in the processes of solidification and immobilization of ash and dust from municipal and hazardous waste incineration plants: EKOKEM (Finland), SPITTELAU (Vienna), Zurich Power Plant (Switzerland), ZUSOK (Warsaw), EKRO (Boleslaw), EKO-SERW Czajka Wastewater Treatment Plant (Warsaw).

Suggested areas of application for the products are: - road construction (road and construction aggregates); - general and industrial construction (blocks, fittings, concrete products);

- raw meal components for cement plants (substitute of alumina, iron, calcium and silicon);

- inert material for the construction of landfills (seals, slope profiling, reclamation).

\subsection{EnviroMIX technology}

EnviroMIX technology is dedicated to the disposal of soil contaminations, sludge, waste containing organic compounds and dangerous substances (heavy metals, halogen compounds, pesticides, PAH cyanides, etc.).

The EnviroMix ${ }^{\circledR}$ process is recognized as leading way of binding and stabilizing many types of hazardous waste and is a breakthrough in the field of neutralization. Stabilization of hazardous waste with using this technology is many times more effective than today's widespread stabilization methods based on the use of Portland cement. The basic distinguishing feature of this technology is several times less demand for stabilizing material with respect to the unit weight of waste. Due to the specificity of bonding of pollutants by magnesium compounds, Enviro technology is resistant to the leaching of the pollutions, which appear sometimes in cement stabilized waste.

The EnviroMix ${ }^{\circledR}$ process is classified as a chemical bonding and solidification (CFS) technology. It is a magnesium oxide based material prepared in a patented dolomite or magnesite process with a high content of magnesium carbonate. The resulting material is crushed, suitably calcined (roasted) and processed according to the individual waste stream of waste disposed. The process is based on the pollutions immobilization as thermodynamically stable mineral phases and the microencapsulation of organic pollutants into mineral structures. Machining can be carried out using commercially available mixing equipment or a suitably designed and constructed mobile installation. It is also possible to modify existing industrial applications to adapt them to stringent health and safety requirements for toxic substances. Stabilized waste could also be used as construction aggregate due to its good physical properties. Process wastes can also be used at location, eg for leveling and landfill. It is important that the waste does not have to be stored at a hazardous waste site anymore, which avoids very high costs for such storage.

The manufacturer gives many benefits of using Enviro Mix ${ }^{\circledR}$. One of them is the possibility of using it directly at the location or storing it into lower class landfills (generally a relatively lower fee applies). Cost reduction is also achieved through favorable mixing ratios in comparition to other methods. The resulting waste could be further mixed with salted or contaminated waters. It is worth noting that the using of Enviromix $\mathbb{R}$ is possible for dispose of waste with high moisture content $(>70 \%)$, reducing the need for expensive and time-consuming decanting (precipitation) process. Liquid waste and sludge are solidified while clays, oils and organic waste are bound.

Enviromix ${ }^{\circledR}$ technology uses conventional mixing techniques and equipment. That is why raw materials could be delivered to in situ disposal sites while allowing safe transport of cleaned materials.

This method could be used for the disposal of mixed waste with a high concentration of organic and inorganic substances.

\subsection{SULTECH technology}

Original technical and technological solution patented for the recovery of hazardous industrial waste containing heavy metals in solid form (powders, granules, etc.) by stabilizing them with sulfur polymer in SULTECH ${ }^{\circledR}$ polymer concrete. The process of stabilizing and recovering hazardous industrial waste in polymer concrete is particularly suitable for stabilizing waste which contain heavy metals such as cadmium, chromium, barium, zinc, copper, calcium, iron, nickel, arsenic, manganese, lead, aluminum, magnesium, ashes from waste incineration plants. Solidification of waste, especially hazardous containing heavy metals, consists in forming with sulfur polymer sulphides of these metals that are insoluble in water. The received SULTECH® Polymer Concrete containing waste is an inert and excellent thermoplastic material (it can be processed many times), from which it could be formed many of very good products for excample: drainage lines, $\mathrm{NOE} \circledast$ flood disks, sewerage wells, road boards, road and motorway components, railroad weights e.t.c. Polymer Concrete SULTECH ${ }^{\circledR}$ has the following properties:

- very good mechanical properties (high compressive strength and flexural strength), which exceeds similar Portland concrete;

- resistance to aggressive media including: acids, salts, seawater;

- very low absorption;

- tightness and frost resistance;

- short forming time;

- short time to achieve nominal strength;

- waste of production; 
- comparable coefficient of expansion with Portland cement;

- recyclable products (thermoplastic).

\section{Summary}

Due to the large diversity of hazardous waste, it is difficult to distinguish one, the best method of their immobilization. It should be considered that the used technology have to be tailored to the investor needs. An important issue is the physical characteristics of waste at entry. It determines the importance of choosing the right technology.

The application areas of each of the presented methods are relatively narrow, so it is extremely important to precisely know what kind of waste we are dealing with, and what are the real possibilities of its immobilization.

Another determinant factor from the waste generator point is the processing unit cost over a ton of the material. It forces the development of new, more efficient disposal methods and continually checking the effectiveness of the currently methods, their optimization and improvement to maintain satisfactory results.

\section{References}

1. S. Caijun, A. Fernandez- Jimenez, J. Hazard. Mater. 173 (2006)

2. T. Marcinowski, K. Banaszkiewicz, Research on the mechanical durability and chemical stability of soldifier hazardous waste (Environmental engineering III, 2010)

3. V. Misra, S.D. Pandey, Environment Intermational, 31 (2005)

4. J. Pyssa, Gospodarka Surowcami Mineralnymi, 24 (2008)

5. M.A. Tantaway, A.M. El-Roudi, A.A. Constr. Build. Mater., 30 (2012)

6. W. Wasiak, S. Różalski, Przegląd Komunalny, 6 (2003) 